\title{
DIPOLE-LIKE NEURONAL SOURCES OF THETA RHYTHM IN DORSAL HIPPOCAMPUS, DENTATE GYRUS AND CINGULATE CORTEX OF THE URETHANE-ANESTHETIZED RAT
}

\author{
B.W.A. FEENSTRA ${ }^{1}$ and J. HOLSHEIMER ${ }^{2}$ \\ Bio-information Group, Department of Electrical Engineering, Twente University of Technology, Enschede (The \\ Netherlands)
}

(Accepted for publication: February 7, 1979)

Theta activity in the hippocampus was first investigated by Green and Arduini in 1954. Since then much has been learned about the localization of the neuronal sources of theta activity in the brain. Nevertheless, the relations between theta field potentials measured at different sites in the brain, including areas outside the hippocampus, have not yet been sufficiently explained in terms of their neuronal sources.

The laminar distribution of theta field potentials in the rat hippocampus has been investigated by several authors (Winson 1974, 1976a; Bland and Whishaw 1976). In the freely moving rat, maxima of theta amplitude were found approximately at the level of the stratum pyramidale of CA1 and in the dorsal blade of the dentate area. Between these peaks a gradual phase shift of $180^{\circ}$ is found in the stratum radiatum (Winson 1974). Urethane anesthesia does not change this distribution pattern (Bland and Whishaw 1976) but after curare injection there is only one amplitude maximum in the vicinity of the hippocampal fissure and a sudden phase reversal occurring at the level of the stratum radiatum of CA1 (Winson 1976a). The latter distribution pattern has also been found in

\footnotetext{
1 Present address: Department of Medical and Physiological Physics, Physics Laboratory, State University, Princetonplein 5, Utrecht, The Netherlands.

${ }^{2}$ Reprint requests to J. Holsheimer, Dept. of Electrical Engineering, Twente University of Technology, P.O. Box 217,7500 AE Enschede, The Netherlands.
}

the freely moving rabbit (Winson $1976 \mathrm{~b}$ ) and in the rabbit after application of curare (Green et al. 1960) or under urethane anesthesia (Bland et al. 1975). Although the laminar distributions of theta rhythm may vary under different conditions and in different species (i.e. rat and rabbit), the ways in which the field potentials in the hippocampal complex are produced by their neuronal sources are considered to be the same. Two different concepts have been proposed, both starting from the principle that theta activity arises as a result of synchronous postsynaptic potentials in one or more populations of neurons in the hippocampal formation (consisting of the hippocampus proper and the dentate area). Winson $(1974,1976 a$ and $b)$ and Bland et al. $(1975,1976)$ have suggested that the hippocampal and dentate theta activities are the result of two closely coupled generators, approximately $180^{\circ}$ out of phase. They based this conclusion on different findings: (1) on either side of the phase reversal in the stratum radiatum the theta rhythms have different amplitude modulations and generally dissimilar wave shapes (Winson 1974); (2) firing of CA1 pyramidal cells and dentate granule cells is rhythmically coupled with the theta activity (Bland and Whishaw 1976).

On the other hand, Green et al. (1960) and Artemenko (1972) have stated that one generator in the CA1 field with a dipole-like character could produce this theta activity pattern in the rabbit. Their conclusion was based on the finding of only one amplitude maximum 
occurring in the distal part of the stratum radiatum near the fissure, and the concept of identical source-sink configurations of a population of CA1 pyramidal cells giving rise to this dipole-like extracellular field. Because no second reversal was noted in the layer of dentate granule cells, Green et al. (1960) concluded that there was no theta generator in this structure. The question therefore remains of whether a phase reversal of theta activity in the dentate area exists.

Theta activity in parts of the neocortex overlying the hippocampus has been considered to be solely the consequence of volume conduction from the underlying hippocampal sources (Artemenko 1972; Winson 1974; Bland et al. 1975; Gerbrandt et al. 1978) although theta activity has been recorded in parts of the rabbit neocortex not overlying the hippocampus (Efremova and Trush 1973) and in cat neocortex (Creutzfeldt and Meisch 1963). This study - published in a preliminary form (Holsheimer and Feenstra 1977b) - presents evidence in favor of the existence of dipole-like theta sources both in CA1 and in the dorsal granular cell layer, as well as in the cingulate neocortex; in all these cases phase-reversal at the site of an amplitude minimum is found.

\section{Material and methods}

Nine female Wistar rats $(210-250 \mathrm{~g})$ under light urethane anesthesia $(1.1-1.4 \mathrm{~g} / \mathrm{kg})$ were used. Field potentials were recorded simultaneously with a fixed and a moving electrode in the hippocampus, fascia dentata and neocortex against a reference earth electrode (the ear plugs and nose piece of the stereotactic apparatus). In these brain structures theta activity was encountered either spontaneously or following tactile stimulation, e.g. gently stroking the rat's fur.

One electrode was fixed within the dorsal hippocampus in order to monitor theta rhythm continuously. The field potential at various sites within and outside the hippocam- pus was recorded by means of the moving electrode and related in amplitude and phase to the signal recorded by way of the fixed electrode.

Glass-coated platinum semi-microelectrodes $(20-50 \mu \mathrm{m})$ were used for recording. The two electrodes were attached to the stereotactic apparatus with microdrives at an angle of $17.5^{\circ}$. During the experiments, an electronically controlled heating coil was used to maintain a constant body temperature $\left(38^{\circ} \mathrm{C}\right)$ in order to prevent temperature changes affecting theta activity (Whishaw and Vanderwolf 1971). Series of records were obtained while the moving electrode penetrated the brain step by step.

Taking into account the stochastic nature of the field potentials, power spectra, squared coherence and phase spectra of the two signals were calculated on a digital computer (DEC, PDP-11/40 system). Selected epochs of $8 \mathrm{sec}$ were sampled at a frequency of 128 $\mathrm{c} / \mathrm{sec}$ after anti-aliasing filtering at $32 \mathrm{c} / \mathrm{sec}$. A Papoulis window was used for frequency averaging. Laminar profiles of the phase and the squared coherence along the moving electrode trajectory were constructed from the calculated spectral functions for the theta peak frequency and its second harmonic. Amplitude ratio profiles were determined from the root of the quotient of the power spectra of the moving and the fixed electrode signals at the theta peak frequency.

The trajectories of the electrodes were worked out beforehand with the aid of a brain atlas (de Groot 1959) and after the experiments they were verified histologically after staining $10 \mu \mathrm{m}$ serial sections using the Klüver method. We also used the occurrence of spike activity for the localization of the pyramidal and granular layers on the electrode trajectories.

\section{Results}

Spontaneous theta activity with a peak in the power spectrum between 4.0 and 5.0 

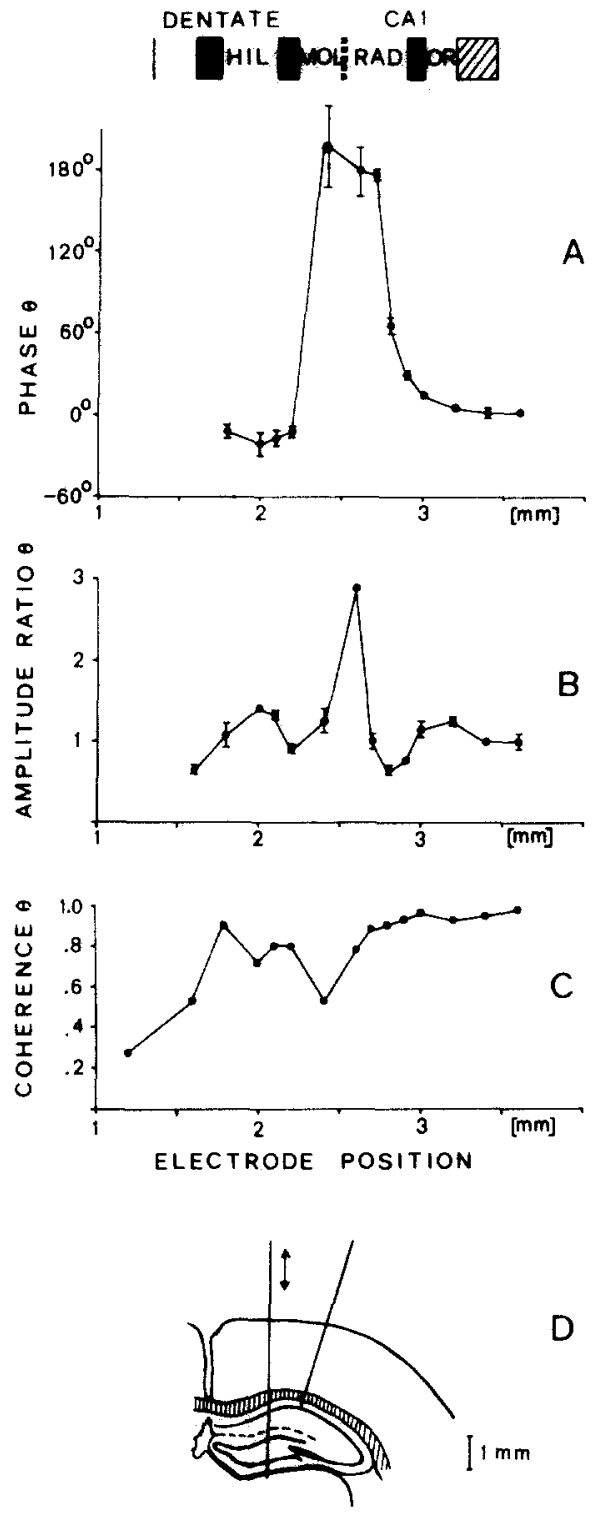

Fig. 1. Phase (A), amplitude ratio (B) and squared coherence $(C)$ profiles at theta peak frequency (4.5$5 \mathrm{c} / \mathrm{sec}$ ); mean and standard deviation of 3 signal epochs; horizontal axis indicates the position of the moving electrode. $\mathrm{D}$ : reconstruction of electrode trajectory; moving electrode is indicated by double arrow; fixed electrode is in str. oriens. HIL, hilus; MOL, dentate molecular layer; $R A D$, stratum radiatum of $\mathrm{CA} 1$; OR, stratum oriens of $\mathrm{CA} 1$; black, layers of neuronal somata; shaded, white matter between CA1 and neocortex; dashed line, hippocampal fissure. $\mathrm{c} / \mathrm{sec}$ was found in large parts of the hippocampus proper, the dentate gyrus and the overlying neocortex. Recordings in the CA1 field and dentate gyrus also showed large

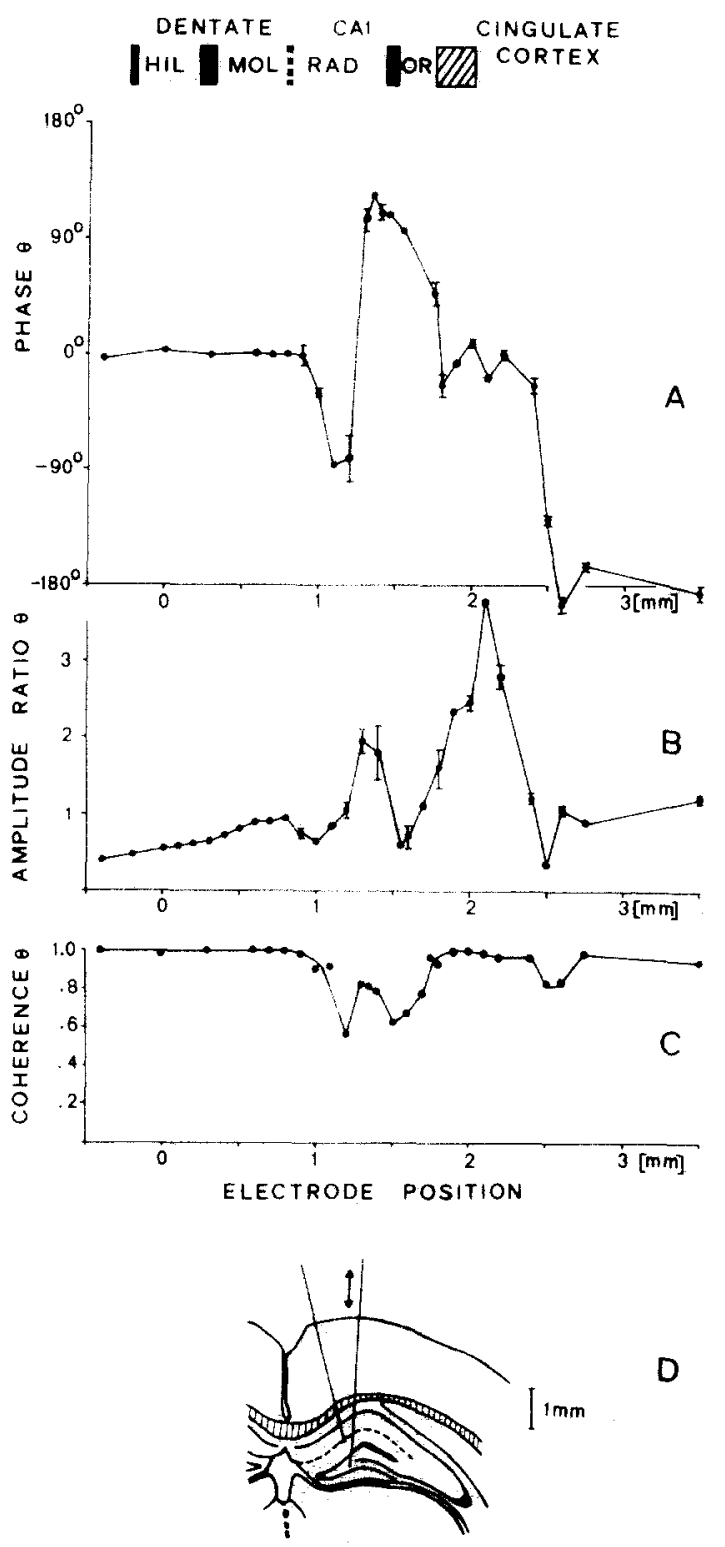

Fig. 2. Another example of phase (A), amplitude ratio (B) and squared coherence (C) profiles at theta peak frequency $(4.5-5 \mathrm{c} / \mathrm{sec})$. $\mathrm{D}$ : reconstruction of electrode trajectory; moving electrode is indicated by double arrow; fixed electrode in molecular layer. Abbreviations as in Fig. 1. 
amplitude beta activity with most of its power between 15 and $55 \mathrm{c} / \mathrm{sec}$, superimposed on the theta activity.

The squared coherence at the theta peak frequency of all the paired recorded signals in the hippocampal formation, as well as in the overlying neocortex, differed significantly from zero $(P<0.025)$. Hence theta activities in these structures were linearly related. The squared coherence profiles had values above 0.7 at nearly all positions (Figs. $1 \mathrm{C}$ and $2 \mathrm{C}$ ).

Because of this linear relationship between theta activities in CA1, dentate gyrus and neocortex, phase relations with low variance could be found. Phase profiles showed gradual shifts of approximately $180^{\circ}$ in the hippocampus and in the cingulate (medial) cortex over a distance of $0.1-0.4 \mathrm{~mm}$ perpendicular to the cell layers. These phase reversals were found in: (1) the proximal part of the stratum radiatum of the CA1 field; (2) the proximal part of the molecular layer of the dorsal dentate gyrus; (3) layer V or VI of the cingulate cortex (Zeman and Innes 1963) (Figs. 1A and 2A).

Phase reversals were found in CA1 in all experiments, but in the dentate and neocortex only in some of the experiments. We still found a peak in the amplitude in the deep layers in some cingulate cortical laminar profiles, without a phase reversal. We also found smaller phase shifts at the borders between the cingulate cortex, the hippocampus proper and the dentate area (Fig. 2A).

The amplitude ratio profiles tended to have low values in the phase shift regions. This is obvious in Fig. 1B, which shows a maximum near the fissure and low values in the phase reversal regions in $\mathrm{CA} 1$ and in the granular layer. Fig. 2B shows clear minima in the phase reversal regions in $\mathrm{CA} 1$ and in the cingulate cortex, and maxima in the cingulate cortex, in CA1 near the pyramidal layer and-less clearly - in the molecular layer of the dorsal dentate area. The reconstructed electrode trajectories corresponding to the profiles discussed above are shown in Figs. 1D and 2D.

From the estimates of the spectral func-

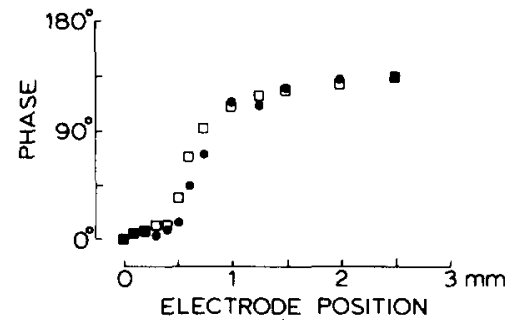

Fig. 3. Phase profile at theta peak frequency (squares) and its second harmonic (circles). Horizontal axis indicates the position of the moving electrode. Phase jump in CA1.

tions from electrode trajectories in CA1 a high coherence at twice the theta frequency was found. This permitted us to calculate low variance phase profiles for the theta frequency as well as for its second harmonic. Fig. 3 shows the phase profiles for both the theta frequency and its second harmonic, and clearly demonstrates that the two profiles resemble each other very closely.

\section{Discussion}

Hippocampal and dentate theta activity

The present results differ in some aspects from the results of Winson (1974) and Bland and Whishaw (1976), although in general terms they confirm their conclusions. We also found two amplitude peaks in the laminar profiles, one near the hippocampal fissure and one near the dorsal CA1 pyramidal layer and a $180^{\circ}$ phase shift in a region of $0.18-0.35$ $\mathrm{mm}$ dorsal to the fissure in the stratum radiatum. A theta phase reversal in the dorsal molecular layer of the dentate area, however, has not previously been reported. We also ascertained a high coherence, i.e. a clear linear relationship, between the theta activities in CA1 and in the dentate area. These results can draw the two different concepts about theta generation, mentioned in the introduction, into one model of the sources of hippocampal theta activity. This will be discussed later. Moreover, we present another new finding: that a neocortical theta source exists. 


\section{Neocortical theta activity}

Artemenko (1972), Winson (1974), Bland and Whishaw (1976) and Gerbrandt et al. (1978) consider neocortical theta field potentials to be the result of volume conduction from the underlying hippocampal sources. Indeed, a linear relationship between hippocampal and neocortical theta has also been found by us. Nevertheless, we found distinct phase reversals and changes in amplitude in the cingulate cortex (Fig. 2). We therefore conclude that, in this part of the neocortex in the urethane-anesthetized rat, the main sources of cortical theta activity are within the neocortex, since volume conduction from the hippocampus will not produce phase shifts nor an increasing amplitude with increasing distance from the source (Holsheimer and Feenstra 1977a). Petsche and Stumpf (1960) found a 'loose phase coupling' between neocortical and hippocampal theta rhythms, and during a hypersynchronized arousal pattern they found regular theta rhythm in the hippocampus, but irregular theta activity in the neocortex of the awake rabbit. Winson (1974) sometimes found intermittent or even absence of theta activity in neocortex during regular hippocampal theta activity in the awake rat. These results also suggest that neocortical theta activity should not be considered to be solely the result of volume conduction from the hippocampus. Efremova and Trush (1973) recorded closely coupled theta activity in motor, visual and auditory cortical areas of the rabbit during a conditioned reflex. Since theta activity in the visual and auditory cortex is hard to explain as resulting from volume conduction from the hippocampus (Bland and Whishaw 1976; Gerbrandt et al. 1978 ), these results also suggest theta sources in parts of the neocortex. Creutzfeldt and Meisch (1963) found spike bursts from pyramidal cells in the somatosensory cortex of the cat during some stage of hypoglycemia, which had a triggered correlation with theta activity in the surface EEG (approx. $5 \mathrm{c} / \mathrm{sec}$ ). From the results of Creutzfeldt and Meisch (1963) and Efremova and Trush (1973) no conclusions can be drawn regarding relations between neocortical and hippocampal theta activity. We conclude that depending on the experimental conditions, neocortical theta activity is mainly caused by neuronal sources within parts of the neocortex.

In order to calculate the laminar profiles, we needed a theta field potential originating from only one site for comparison with the field potential from the moving electrode. Because we were interested primarily in hippocampal theta rhythm, the fixed electrode was placed within the hippocampus, even when part of the electrode trajectory was outside this structure, i.e. in the cingulate cortex.

\section{Neuronal sources of theta activity}

Fig. 3 shows that the phase shift at theta peak frequency is accompanied by an equal phase jump at its second harmonic. This makes it unlikely that the observed phase jumps can be explained in terms of neuronal delay times, since these should cause frequency-dependent phase relations. This result leads us to the conclusion that the phase reversal of the theta field potential in the CA1 field, cingulate cortex and dentate gyrus is an indication of local neuronal sources in each of these structures. This dipole-like electrical field results from identical locations of inward and outward currents in synchronously activated neurons in one layer. In CA1 the population of pyramidal cells is generally considered to be the theta source, and in the dentate area it is obviously the population of granular cells (Feder and Ranck 1973; Bland and Whishaw 1976). In the dorsal hippocampus, therefore, there are two linearly coupled sources of theta activity, one in CA1 and one in the dentate area. It seems likely that these sources are activated approximately in phase, instead of alternating with an interval of approximately $100 \mathrm{msec}$ at a theta peak frequency of $5 \mathrm{c} / \mathrm{sec}$. A delay 
time between the activation of the two cell layers would give rise to phase relations which were linearly dependent on the theta peak frequency, in contradiction to the experimental results. We may state, therefore, that in the hippocampus the theta rhythm is generated by two equivalent dipole layers centered at the two sites where phase reversal was encountered, i.e. the stratum radiatum of CA1 and the molecular layer of the dentate, obviously the populations of pyramidal cells and granular cells, respectively. The field potential between the two equivalent dipole layers would be determined by the relative strengths of the two generators as would the location of an amplitude maximum in this area.

Concerning the phase reversal in the cingulate cortex, the theta source might be the population of large pyramidal cells having their somata in layer $V$. We did not find phase reversals of the theta field potential in other fields of the neocortex above the hippocampus. This is in agreement with the results published recently by Gerbrandt et al. (1978), except for the cingulate cortex. They recorded from curarized rats, while we used urethane-anesthetized rats.

Under different experimental conditions, different types of theta field potentials (frequency, amplitude) may be found, possibly resulting from different combinations of neuronal theta sources. In some aspects the urethane-theta activity differs from the behavior-related theta activity, although the hippocampal sources seem to be identical in the rat (Bland and Whishaw 1976) and rabbit (Bland et al. 1975; Winson 1976b).

We may conclude from the high values of coherence at the theta peak frequency that the theta generating cell layers in the CA1 field, dentate area and cingulate cortex have powerful mutual neuronal connections or have a common input signal from a distant theta generator (i.e. the septum), or both.

In order to interpret the hippocampal laminar profiles with regard to the sites of synaptic activation on the soma-dendritic membranes in the respective cell layers, a theoretical analysis of the laminar profiles by computer simulation appears to be necessary (Lopes da Silva and Arnolds 1978). Preliminary results of these simulations for the dorsal hippocampal formation indicate that our interpretation of the laminar profiles by means of two equivalent dipole layers is not inconsistent with the experimental observations.

\section{Summary}

Spatial distribution of theta activity was investigated in the dorsal hippocampal formation and overlying neocortex of the urethaneanesthetized rat. Laminar phase profiles from semi-microelectrode penetrations showed approximately $180^{\circ}$ phase shifts combined with small amplitude values in stratum radiatum of CA1, in stratum moleculare of the dentate gyrus and in layer V/VI of the cingulate cortex at theta peak frequency. Evidence has been presented that layers of neurons in CA1, in the dorsal granular layer and in the cingulate cortex are the sources of dipole-like theta field potentials. A strong linear relationship between the neuronal theta sources in hippocampal $\mathrm{CA1}$, dentate area and cingulate cortex was found.

\section{Résumé}

Sources neuronales, de type dipolaire, du rythme thêta dans l'hippocampe dorsal, le gyrus dentatus et le cortex cingulaire du rat anesthésié à l'uréthane

La distribution spatiale de l'activité thêta a été recherchée dans l'hippocampe dorsal et le cortex susjacent chez le rat anesthésié à l'uréthane. Les profils laminaires, établis à partir de pénétrations de sémi-microélectrodes, ont révélé des glissements de phase de $180^{\circ}$, liés à des amplitudes faibles, dans le stratum radiatum de CA1, le stratum moléculaire du gyrus 
dentatus, et les couches V et VI du cortex cingulaire, pour la fréquence dominante thêta. On prouve que les assises neuronales de CA1, celles de la couche granulaire dorsale, et celles du cortex cingulaire, constituent les sources d'un champ de potentiel thêta en dipole. Une relation linéaire étroite entre les sources neuronales thêta de CA1, du gyrus dentatus et du cortex cingulaire a été établie.

We are grateful to Dr. F.C. Kuipers and his staff of the Regional Laboratory for Pathology in Enschede, The Netherlands for preparing the serial sections, to Dr. F.H. Lopes da Silva for suggestions and discussions, to $\mathrm{Dr}$. A. Crowe for correcting the manuscript, and to Miss G.G.M. Steijlen for typing the manuscript.

\section{References}

Artemenko, D.P. Role of hippocampal neurons in theta wave generation. Neurofiziologiya, 1972, 4: 409-415 (translated edition).

Bland, B.H., Andersen, P. and Ganes, T. Two generators of hippocampal theta activity in rabbits. Brain Res., 1975, 94: 199-218.

Bland, B.H. and Whishaw, I.Q. Generators and topography of hippocampal theta (RSA) in the anaesthetized and freely moving rat. Brain Res., 1976, 118: $259-280$.

Creutzfeldt, $O$. and Meisch, J.J. Changes of cortical neuronal activity and EEG during hypoglycemia. Electroenceph. clin. Neurophysiol., 1963, Suppl. 24: 154-171.

Efremova, T.M. and Trush, V.D. Power spectra of cortical electric activity in the rabbit in relation to conditioned reflexes. Acta Neurobiol. Exp., 1973, 33: $743-755$.

Feder, R. and Ranck Jr., J.B. Studies on single neurons in dorsal hippocampal formation and septum in unrestrained rats. Part II. Hippocampal slow waves and theta cell firing during bar pressing and other behaviors. Exp. Neurol., 1973, 41: $532-555$.
Gerbrandt, L.K., Lawrence, J.C., Eckhardt, M.J. and Lloyd, R.L. Origin of the neocortically monitored theta rhythm in the curarized rat. Electroenceph. Clin. Neurophysiol., 1978, 45:454-467.

Green, J.D. and Arduini, A.A. Hippocampal electricai activity in arousal. J. Neurophysiol., 1954, 17 : $533-557$.

Green, J.D., Maxwell, D.S., Schindler, W.J. and Stumpf, C. Rabbit EEG 'theta' rhythm: its anatomical source and relation to activity in single neurons. J. Neurophysiol., 1960, 23: 403-420.

Groot, J. de The rat forebrain in stereotaxic coordinates. Verh. Kon. Ned. Ac. v. Wet., tweede reeks, 1959, $7: 1-40$.

Holsheimer, J. and Feenstra, B.W.A. Volume conduc. tion and EEG measurements within the brain: a quantitative approach to the influence of electrical spread on the linear relationship of activity measured at different locations. Electroenceph. Clin. Neurophysiol., 1977a, 43:52-58.

Holsheimer, J. and Feenstra, B.W.A. Neuronal sources of theta waves in hippocampus and neocortex and their relations in the urethane anesthetized rat. Electroenceph. Clin. Neurophysiol., 1977b, 43: 573.

Lopes da Silva, F.H. and Arnolds, D.E.A.T. Physiology of the hippocampus and related structures. Ann. Rev. Physiol., 1978, 40: 185-216.

Petsche, H. and Stumpf, Ch. Topographic and toposcopic study of origin and spread of the regular synchronized arousal pattern in the rabbit. Electroenceph. Clin. Neurophysiol., 1960, 12: 589600 .

Winson, J. Patterns of hippocampal theta rhythm in the freely moving rat. Electroenceph. Clin. Neurophysiol., 1974, 36: 291-301.

Winson, J. Hippocampal theta rhythm I. Depth profiles in the curarized rat. Brain Res., 1976a, 103: $57-70$.

Winson, J. Hippocampal theta rhythm II. Depth profiles in the freely moving rabbit. Brain Res., 1976b, 103: 71-79.

Whishaw, I.Q. and Vanderwolf, C.H. Hippocampal EEG and behaviour: effects of variation of body temperature and relation of EEG to vibrissae movement, swimming and shivering. Physiol. Behav., 1971, 6: 391-397.

Zeman, W. and Innes, J.R.M. Craigie's Neuroanatomy of the Rat. Academic Press, New York, 1963. 